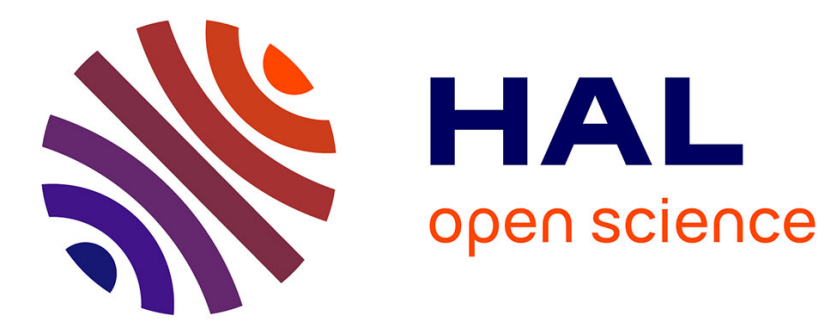

\title{
Regular BIE for three-dimensional cracks in elastodynamics
}

\author{
Marc Bonnet, Huy Duong Bui
}

\section{To cite this version:}

Marc Bonnet, Huy Duong Bui. Regular BIE for three-dimensional cracks in elastodynamics. IUTAM Symposium, 1987, San Antonio, United States. pp.41-48, 10.1007/978-3-642-83003-7_5 . hal00122005

\section{HAL Id: hal-00122005 \\ https://hal.science/hal-00122005}

Submitted on 3 Jan 2022

HAL is a multi-disciplinary open access archive for the deposit and dissemination of scientific research documents, whether they are published or not. The documents may come from teaching and research institutions in France or abroad, or from public or private research centers.
L'archive ouverte pluridisciplinaire HAL, est destinée au dépôt et à la diffusion de documents scientifiques de niveau recherche, publiés ou non, émanant des établissements d'enseignement et de recherche français ou étrangers, des laboratoires publics ou privés.

\section{다)(1) $(5$}

Distributed under a Creative Commons Attribution - NonCommerciall 4.0 International 


\title{
Regular B.I.E. for Three-Dimensional Cracks in Elastodynamics
}

\author{
M. BONNET and H. D. BUI \\ Electricité de France, Dept.MMN, Clamart and \\ Ecole Polytechnique, 91128 Palaiseau, France
}

$\underline{\text { Summary }}$

In this paper, a regular B.I.E is proposed for 3D curved cracks in elastodynamics. We first derive the singular integral equations for smooth cracks as well as for kinked and crossed cracks, which generalize the B.I.E given by several authors. Then we apply the regularization technique to the equations.

\section{Int roduction}

The difficulty in solving 3D crack problem using B.I.E methods have been recognized in the past [1],[2],[3]. It might be explained by the conjunction of two serious mathematical difficulties : the singularities of the kernels which result in Cauchy principal value integrals, and the high variation of the crack displacement near the crack front.

To overcome these difficulties, polch et al.[4] proposed the improvement of the interpolation procedure, using for instance quadratic 8-nodes elements. lowever, considering mathematical formulations of B.I.E which use systematically non singular kernels and which we call the regularization technique appears to be a better approach. A review of methods of regularizations of integrals can be found in [5]. In recent works [6], [7], [8] accurate results have been obtained by the regularization technique which was combined with an advanced interpolation procedure.

The regularization method was applied to B.I.E for 3D plane cracks [5], [6]. In this paper, we reconsider the 3D curved crack problem which was formulated in terms of singular B.I.E by Levan and Royer [9] in the static case, and by V. Sladek and J. Sladek [11] in the dynamic case. We shall present the regular integral equations, for smooth cracks as well as for kinked and crossed cracks. We will not discuss the numerical aspects of the new equations, which are beyond the scope of the paper. 


\section{Notations}

We consider the problem of a 3D curved crack embedded in an infinite elastic body. The crack surface $S$ is assumed to be sectionally smooth and coincides with the lower face $S^{-}$of the crack, Fig. 1, whose unit normal is $n(x)$. We shall use the same notation for the spatial position of a point and its vector position $\mathrm{x}_{\mathrm{x}} \mathrm{x}_{\mathrm{i}} \mathrm{e}_{\mathrm{i}}\left(\mathrm{e}_{1}, \mathrm{e}_{2}, \mathrm{e}_{3}\right.$ are fixed Cartesian basis vectors). The traction vector on $S$, associated with any continuously differentiable displacement field $u(x)$ in $R^{3}$, is given by $t(x)=F u(x)$, where $F$ denotes the differential operator

$$
\mathrm{F}(\partial \mathrm{x}, \mathrm{n}(\mathrm{x}))=2 \mu \mathrm{n} \cdot{ }^{\dagger} \operatorname{grad}+\lambda \mathrm{ndiv}+\mu \mathrm{n}_{\wedge} \mathrm{Curl}
$$

In the above, ( $\dagger$ ) is the transposition of tensor, $\lambda$ and $\mu$ are the Lame constants. The following notations are also used :

$$
\begin{array}{ll}
\mathrm{C}_{\mathrm{ijk}}=\lambda \delta_{\mathrm{ij}}^{\delta} \mathrm{kl}+\mu\left(\delta_{\mathrm{ik}}^{\delta} \mathrm{jl}^{+\delta} \mathrm{il}^{\delta} \mathrm{jk}\right) & : \text { Elastic moduli tensor } \\
\mathrm{L}^{\omega}=\mu \Delta+(\lambda+\mu) \operatorname{grad} \mathrm{div}+\rho \omega^{2} \mathrm{Id} & \text { : Elastodynamic operator }
\end{array}
$$

We shall consider the Stokes displacement tensor $E^{\omega}(x, y)$ which is the fundamental solution of the operator $\mathrm{L}_{(\mathrm{y})}^{\omega}$ in which differentiations are made with respect to $y$. The case $\omega=0$ corresponds to Kelvin's tensor $E^{0}(x, y)$. We recall that $E_{i ; k}^{\omega}(x, y)$ is the $i$-component of the displacement field at $y$, due to the harmonic point force along $e_{k}$-direction, acting on $x$. The tensor $E^{\omega}$ possesses the well-known symmetry properties $E^{\omega}(x, y)={ }^{\dagger} E^{\omega}(x, y)=E^{\omega}(y, x)$ and a weak singularity like $\|x-y\|^{-1}$ when $x \rightarrow y$. A stronger singularity is found in the fundamental traction tensor $T^{\omega}(x, y, n(y))=F(\partial y, n(y)) E^{\omega}(x, y)$, which behaves like the tensor $\mathrm{T}^{\mathrm{O}}$ as $\|\mathrm{x}-\mathrm{y}\|^{-2}$, or more precisely $\left\|\mathrm{T}^{\omega}-\mathrm{T}^{\mathrm{O}}\right\|=0\left(\omega^{2}\right)$ as $x \rightarrow y$. Introducing the stress tensor $\Sigma^{\omega}$, one can write $T^{\omega}$ as follows

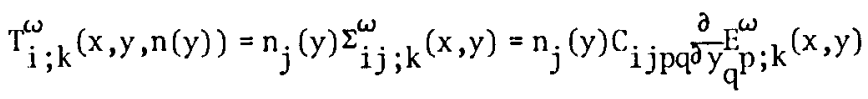

\section{Tangential operators}

To reduce the singularities of kernels, we are led to perform an integration by parts of integrals over curved surfaces. In order to do so, several mathematical identities using tangential operators are required. Consider first a field $f(x)$ function of points $x$ on $S$. The function $f$ is not defined outside $S$, as illustrated by the following examples $f=\Phi_{i}(x)=u_{i}\left(x^{+}\right)-u_{i}\left(x^{-}\right)$, 
i.e the crack displacement discontinuity, and $f=n_{i}(x)$. Therefore the derivatives $f_{, j}$ are meaningless, unless $e_{j}$ are the basis vectors of the plane of a crack. For curved cracks, we have to extend, in some way, the domain of definition of $\mathrm{f}$, for example by introducing the function $\hat{\mathrm{f}}(\mathrm{x})$

$$
\hat{f}(x)=f(P(x)) \quad x \in D, P(x) \in S
$$

where $\mathrm{D}$ is a neigbourhood of $\mathrm{S}, \mathrm{P}(\mathrm{x})$ is the orthogonal projection onto $\mathrm{S}$. Clearly, the restriction of $\hat{f}$ to the surface $S$ is equal to $f$. Moreover the normal derivative of $\hat{\mathrm{P}}$ is equal to zero, i.e the vector $\operatorname{grad} \hat{\mathrm{P}}$ is tangent to $S$; therefore it may be used to define the tangential $\operatorname{gradient}_{\operatorname{grad}} \mathrm{f}$ of the function $f(x)$. For the consistency of notations, we put $\operatorname{grad}_{S}=\operatorname{grad}-\mathrm{na} / \partial \mathrm{n}$. Thus $\operatorname{grad}_{S} \hat{f}=\operatorname{grad} \hat{\mathrm{f}}=\operatorname{grad}_{S} \hat{\mathrm{f}}$. Another tangential operator, $V^{y}=n(y){ }_{\wedge} \operatorname{grad}$, possesses the property

$$
\int_{S} n_{\wedge} \operatorname{grad} \hat{\mathrm{f}} \mathrm{dS}=\int_{\partial S} f(y) \tau(y) d s_{y}
$$

where $\tau$ is the unit tangent vector to $\partial S$. In the following, unless stated otherwise, we shall omit the symbol $\left({ }^{\wedge}\right)$ keeping in mind when necessary the extension (3). The j-component of $v^{y} f$ then reads

$$
\left(V^{y}\right)_{j}=\epsilon_{j p q} V_{p q}^{y} f(y) \quad, \quad v_{p q}^{y}=n_{p} \frac{\partial}{\partial y_{q}}-n_{q} \frac{\partial}{\partial y} y_{p}
$$

In some applications, $f=\Phi_{i}$, the $i$-component of the crack discontinuity being equal to zero along the crack border, the line integral in Eq. (4) vanishes identically. For the kinked crack, I:q. (4) applies to each domain $S_{1}$ or $S_{2}$. The line integral term also vanishes because the contributions to the line integrals, along the common boundary $\Gamma$ of $S_{1}$ and $S_{2}$, are of opposite sign, due to the continuity of $\Phi_{i}$ across $\Gamma$. For the crossed cracks, the line integral does not vanish, as observed by Levan and Royer [9] in their quasi static analysis of curved cracks.

\section{Singular B.I.E for curved cracks}

Our aim here is not to repeat in full details the derivation of the singular B.I.I:, but rather to outline some key steps of the method of derivation used in [9], [11], which we generalize for a complex geometry of cracks in elastodynamics. Classically, the perturbation field $u(x)$ caused by the crack which receives the incident wave $\mathrm{u}^{\text {inc }}(\mathrm{x}), x \in \mathrm{R}^{3}$, can be expressed in terms of the crack discontinuity as follows 


$$
u_{k}(x)=\int_{S} \Phi_{i}(y) T_{i ; k}^{\omega}(x, y, n(y)) d S_{y} \quad x \in R^{3}
$$

The boundary condition on the crack is $t(x)=-F(\partial x, n(x)) u^{i n c}(x)$. Formally, the traction vector

$$
t_{1}(x)=-C_{1 p k r} n_{p}(x) \int_{S} \Phi_{i}(y) n_{s}(y) \frac{\partial}{\partial y} \Sigma_{r}^{\omega} \Sigma_{i s ; k}^{\omega}(x, y) d S_{y}
$$

is expressed by a hypersingular integral, with a singular kernel of order $\|x-y\|^{-3}$. To avoid a divergent integral, we consider as the first step, that $x$ belongs to $D$, but not to $S$, and we replace $n(x)$ by $\hat{n}(x)$. In the second step we integrate by parts, using Eq. (4) and setting $\mathrm{f}=\hat{\mathrm{p}}_{\mathrm{i}} \Sigma_{\mathrm{is} ; \mathrm{k}}^{\omega}$. In the last step, we investigate the limit of the integral as $x \rightarrow P(x)$. These steps lead to the Cauchy principal value integrals, which are indicated by the symbol $\left(^{*}\right)$

$$
\begin{aligned}
t_{l}(x)= & C_{1 p k r} n_{p}(x)\left[-\int_{S}^{*} V_{r s}^{y} \Phi_{i}(y) \Sigma_{i s ; k}^{\omega}(x, y) d S_{y}+\right. \\
& \left.+\mu k_{T}^{2} \int_{S} \Phi_{i}(y) n_{r}(y) E_{i ; k}^{\omega}(x, y) d S_{y}\right], x \in S
\end{aligned}
$$

where $\mu \mathrm{k}_{\mathrm{T}}^{2}=\rho \omega^{2}$. Eq. (8) have been derived by J. Sladek and V. Sladek [11] for smooth surface. The above equations hold for the kinked crack, as explained in the preceeding section. When there are more than two crack branches $S_{1}$, $\mathrm{S}_{2}, \mathrm{~S}_{3} \ldots$, a line integral term appears in the right hand side of the above equation and is given by $(x \in S, x \notin \Gamma)$

$$
\{1 \text { ine integral }\}=\sum_{\alpha} \frac{1}{2} C_{1 p k r} n_{p}(x) \int_{\partial S_{\alpha}} \Phi_{i}(y) \Sigma_{i s ; k}^{\omega}(x, y) \tau_{j}(y) \varepsilon_{j r s} d S_{y}
$$

Regular B.I.E for curved cracks

The technique employed in many works is based on the singularity exclusion method. Applying this method to Eq. (8), we obtain

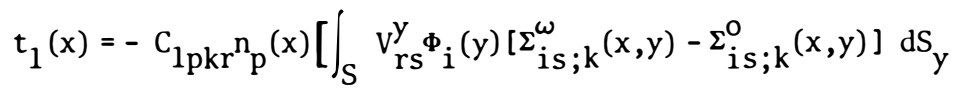

$$
\begin{aligned}
& +\int_{S}\left[V_{r s}^{y} \Phi_{i}(y)-V_{r s}^{x} \Phi_{i}(x)\right] \Sigma_{i s ; k}^{o}(x, y) d S_{y} \\
& -\mu \mathrm{k}_{\mathrm{T}}^{2} \int_{\mathrm{S}} \Phi_{\mathrm{i}}(\mathrm{y}) \mathrm{n}_{\mathrm{r}}(\mathrm{y}) \mathrm{E}_{\mathrm{i} ; \mathrm{k}}^{\omega}(\mathrm{x}, \mathrm{y}) \mathrm{dS} \mathrm{y}_{\mathrm{y}} \\
& \left.+\mathrm{V}_{\mathrm{rs}}^{\mathrm{x}} \Phi_{\mathrm{i}}(\mathrm{x}) \int_{S}^{*} \Sigma_{\mathrm{is} ; \mathrm{k}}^{\mathrm{o}}(\mathrm{x}, \mathrm{y}) \mathrm{dS} \mathrm{y}\right]+ \text { line integral }
\end{aligned}
$$


where the static kernel $\Sigma^{0}$ is used for removing singularities. By virtue of the presence of the term $\Sigma^{\omega}-\Sigma^{\circ}$, the first integrand is not singular, while the second one, if we assume a Hölder condition on the tangential derivatives $V_{r s}^{y} \Phi_{i}(y)$, has a weak integrable singularity. The third integral also converges in the ordinary sense. The fourth integral over each subdomain $S_{\alpha}$ is a Cauchy principal value integral only when $x$ belongs to the same $S_{\alpha}$. In this case, using known values of the components of $\Sigma^{0}$ and taking care of the singular nature of the integral when we perform the integration by parts, we arrive at an expression using regular integrals $\mathrm{I}_{j}$ and $\mathrm{J}_{\text {isk }}$ [8]

$$
\begin{aligned}
& \int_{S_{\alpha}} \Sigma_{i s ; k}^{o} d_{y}=-\frac{1}{4 \pi(\lambda+2 \mu)}\left[\mu\left(\delta{ }_{i k} I_{s}(x)+\delta{ }_{s k} I_{i}(x)+\delta_{i s} I_{k}(x)\right)+\right. \\
& \left.+(\lambda+\mu) \mathrm{J}_{\mathrm{isk}}(\mathrm{x})\right] \\
& I_{i}(x)=\int_{S_{\alpha}} n_{i}(y)\left[\frac{1}{r^{2}} \frac{\partial r}{\partial n(y)}-K(y) \frac{1}{r}\right] d S_{y}-\int_{\partial S_{\alpha}} \frac{1}{r} v_{i}(y) d S_{y} \\
& \mathrm{~J}_{i s k}(x)=\delta_{i k} I_{s}(x)+\delta_{s k} I_{i}(x)+ \\
& +\int_{S}\left\{3 r, i^{r}, s-2 n_{i}(y) n_{s}(y)\right\} n_{k}(y) \frac{1}{r^{2}} \frac{\partial r}{\partial n(y)} d S_{y} \\
& +\int_{S_{\alpha}}^{\alpha}\left\{2 n_{i}(y) n_{s}(y)-r_{, i}{ }^{r}, s\right\} n_{k}(y) K(y) \frac{d S}{r} \quad- \\
& -\int_{S_{\alpha}}^{\alpha}\left\{D_{s}^{y}\left(n_{i} n_{k}\right)+D_{i}^{y}\left(n_{s} n_{k}\right)\right\} \frac{d S}{r} y+ \\
& \left.+\int_{\partial S_{\alpha}} n_{i}(y) n_{k}(y) \dot{v}_{s}(y)+n_{s}(y) n_{k}(y) v_{i}(y)-v_{k}(y) r_{, i}{ }_{, j}\right\} \frac{d s}{r} y
\end{aligned}
$$

In the above, differentiations of $r=\|x-y\|$ are made with respect to $y, K(y)$ stands for the sum of principal curvatures $K=1 / R_{1}+1 / R_{2}$, and $v=n_{\wedge} \tau$. In Eq. (13) we put $D_{i}^{y}=e_{i} \cdot \operatorname{grad}_{S}$. The integrals $I_{i}$ and $J_{i s k}$ are regular because the integrands, as well as the term $r^{-2} \partial r / \partial n$ are of order $0(1 / r)$. Therefore, any classical interpolation procedure, using for instance polar coordinates with the pole $x$, is suitable for the implementation of Eq. (10) in a B.I.E code. Finally, it should be noticed that the line integral term of Eq. (10) is regular for interior points $x \in S_{\alpha}$. With the normal vectors as indicated in Fig. 1, there exists a relation between the crack discontinuities at $\Gamma$,

$$
\Phi_{1}=\Phi_{2}+\Phi_{3}+\cdots
$$

which constitutes, with Eq.(10), the regular B.I.E of 3D crack problem. 
Fundamental t ensors and $\mathrm{k}^{\mathrm{ernel}}$

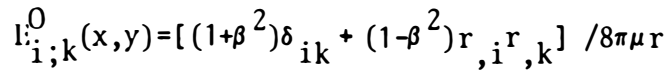

$$
\begin{aligned}
& E_{i ; k}^{\omega}(x, y)=\left[k_{T}^{2} \delta{ }_{i k} G\left(k_{T} r\right)+\left(G\left(k_{T} r\right)-G\left(k_{L} r\right)\right), i k\right] / 4 \pi \mu k_{T}^{2}
\end{aligned}
$$

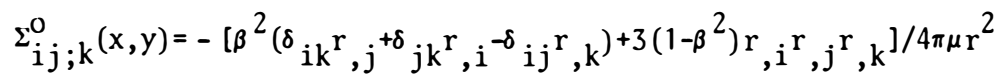

$$
\begin{aligned}
& \Sigma_{i j ; k}^{\omega}(x, y)=\frac{1}{4 \pi} l\left(1-2 \beta^{2}\right) \delta_{i j}\left(G\left(k_{L} r\right)\right), k+\delta_{i k}\left(G\left(k_{T} r\right)\right), j+ \\
& \left.+\delta_{j k}\left(G\left(k_{T} r\right)\right), i+\frac{2}{k_{T}^{2}}\left(G\left(k_{T} r\right)-G\left(k_{L} r\right)\right), i j k\right]
\end{aligned}
$$

where $G(k r)=\exp (i k r) / r, k=\left\{k_{L}, k_{\Gamma}\right\}, k_{\mathrm{T}}^{2}=\omega^{2} \rho / \mu, k_{L}^{2}=\omega^{2} \rho /(\lambda+2 \mu), \beta^{2}=\mu /(\lambda+2 \mu)$.
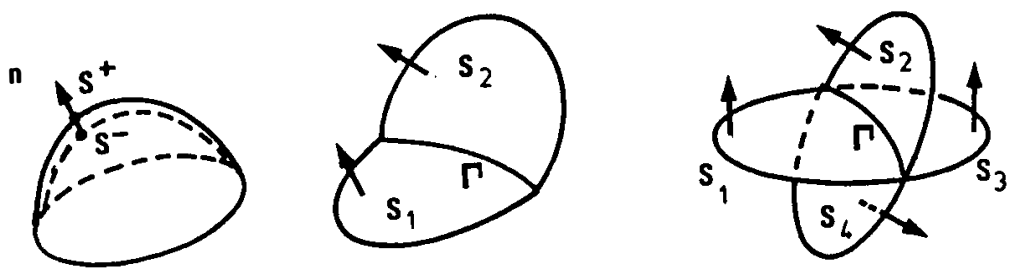

Fig.1. Smooth crack, kinked crack and crossed crack.

\section{References}

1. Bui, H.D.: An integral equation method for solving the problem of plane crack of arbitrary shape. J. Mech. Phys. Solids. 25 (1977) 23-39.

2. Weaver, J. :Three-dimensional crack analysis. Int.J.Solids \& Struct. 13 (1977) $321-330$.

3. Putot, C.: Une nouvelle méthode d'équations intégrales pour certains problèmes de fissures planes. Thèse Univ. Paris 6 (1980) .

4. Polch, E.Z.; Cruse, T.A.; Huang, C.J.B.: Buried crack analysis with an advanced traction B.I.E algoritm. ASME winter annual meeting, (1985), Miami .

5. Bui, H.D.: New trends in B.I.E. methods. Computational Mechanics'86, Tokyo (1986) Springer Verlag, Vol.2, XI.3-8.

6. Polch, E.Z.; Cruse, T.A.; Huang, C.J.B.:Traction B.I.E solutions for flat cracks. Private communication (1986).

7. Rizzo, F.J.; Shippy, D.J.; Rezayat, M.: A boundary integral equation method for radiation and scattering of elastic waves in three dimensions. Int.J.Num.Meth.Engng. 21 (1985) 115-129. 
8. Bonnet, M.: Méthode des équations intégrales régularisées en élastodynamique. Thèse Ecole Nat. des Ponts \& Chaussées, Paris (1986).

9. Levan, A.; Royer, J.: Integral equations for three-dimensional problems. Int. J. Fract. Vol. 31 (1986) 125-142.

10.Sladek, J.; Sladek, V.: Three dimensional curved crack in an elastic body. Int. J. Solids \& Struct. Vol.19 (1983) 425-436.

11.Sladek, J.; Sladek, V.: Dynamic stress intensity factors studied by boundary integro-differential equation. Int. J. Num. Meth. Eng. Vol.23 (1986) 919-928. 